\title{
Experiments using extreme parameters of the NovoFEL radiation
}

\author{
V. V. Kubarev ${ }^{1,2}$, Ya. V. Getmanov ${ }^{1,2}$, O. A. Shevchenko ${ }^{1}$, E. N. Chesnokov ${ }^{3}$, P. V. Ko- \\ shlyakov $^{3}$, L. N. Krasnoperov ${ }^{4}$
}

\author{
${ }^{1}$ Budker Institute of Nuclear Physics, Novosibirsk, Russia, V.V.Kubarev@inp.nsk.su \\ ${ }^{2}$ Novosibirsk State University, Novosibirsk, Russia \\ ${ }^{3}$ Voevodsky Institute of Chemical Kinetics and Combustion, Novosibirsk, Russia
}

${ }^{4}$ NJIT, New Jersey, USA

Three different types of experimental investigations using unique extreme NovoFEL parameters [1] are presented in the report. The first one is continuous terahertz laser discharge which was developed from an effective demonstration of the NovoFEL power [2] to detailed investigation of its physical process [3-5] and creation of a point-like atmospheric-pressure discharge with record temperature of $28000{ }^{\circ} \mathrm{K}$ at plasma density of $2 \cdot 10^{17} \mathrm{~cm}^{-3}$ [6](Fig.1-2).

Second set of the experiments is ultrafast timedomain spectroscopy [7-18] used now both for a single-pulse diagnostics of the NovoFEL radiation [11] and in free-induction-decay molecular spectroscopy including a magnetic spectroscopy $[15,17]$ (Fig. 3) and spectral "cinema" of the generation/decay dynamics of shot-lived molecular OH-radicals (Fig. 4).

Third type of the experiments is high-resolution spectroscopy based on very good coherency between NovoFEL pulses and very narrow lines of the fine structure of its radiation [19,20] (Fig. 5). One-mode filtration by a set of three resonance Fabry-Perot interferometers allows creation a source of powerful ultramonochromatic tunable radiation for highresolution molecular spectroscopy [20] (Fig.6).

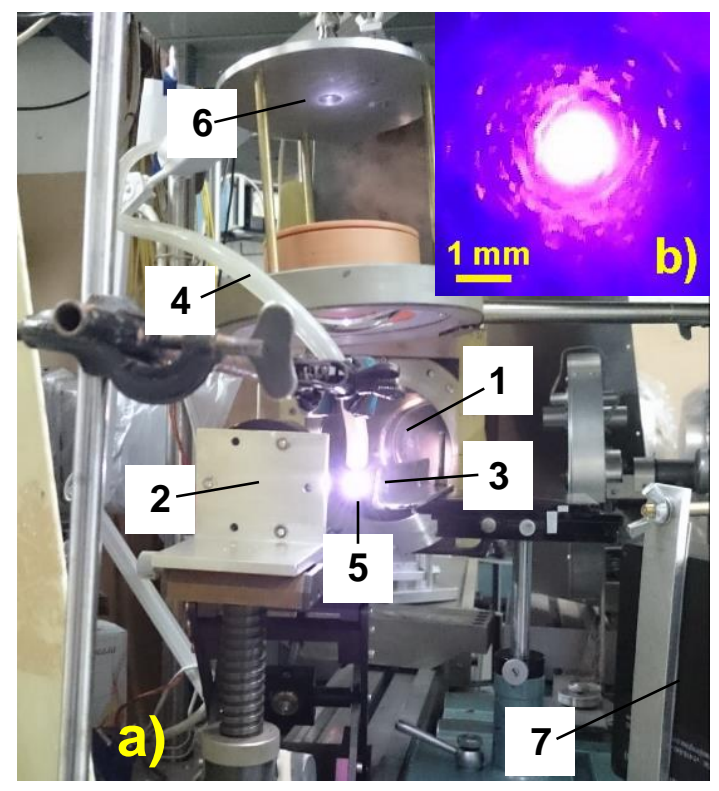

Fig. 1. Experimental setup for investigation of the THz laser discharge (a): 1 - output window of the NovoFEL optical beamline, 2 - main parabolic focusing mirror, 3 - stabilizing spherical mirror, 4 - gas tube, 5 - optical discharge, 6 ultrafast optical photodiode, 7 - Mightex optical spectrometer. Insert (b) - photo of the point-like high-temperature laser discharge through a navy-blue glass filter

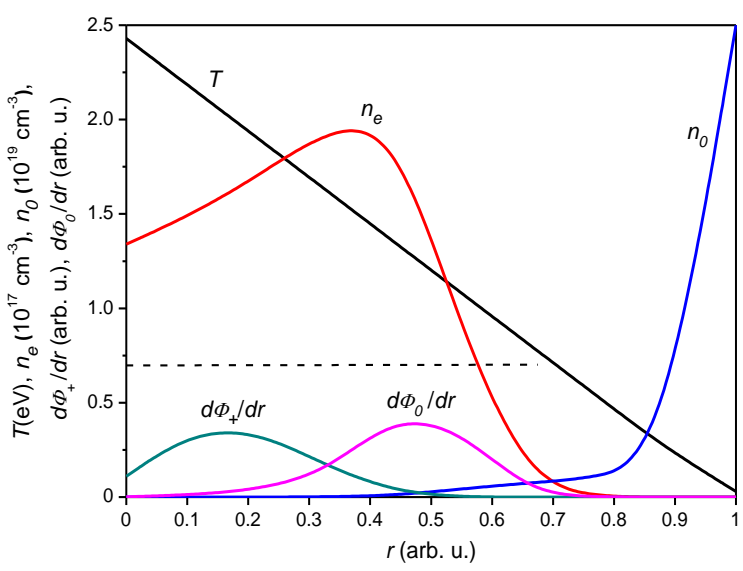

Fig. 2. Radial distributions of temperature $T$, plasma and gas densities $n_{\mathrm{e}}$ and $n_{0}$, and integral radiation of radial spherical layers for ionic $d \Phi_{+} / d r$ and atomic $d \Phi_{0} / d r$ lines of argon in the point-like high-temperature laser discharge. The dashed line indicates the critical plasma density (boundary of reflection of $\mathrm{THz}$ radiation from plasma)

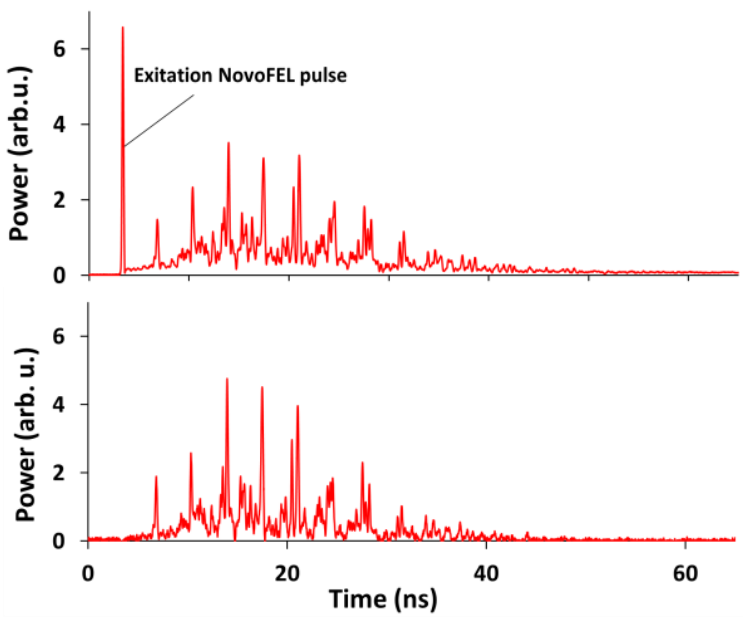

Fig. 3. Polarization separation of powerful excitation NovoFEL pulse and week FID signal of $\mathrm{NO}_{2}$ molecules in magnetic field: upper - slightly misaligned cross input and output polarizers, lower - exactly aligned cross input and output polarizers (excitation pick is fully dumped) 


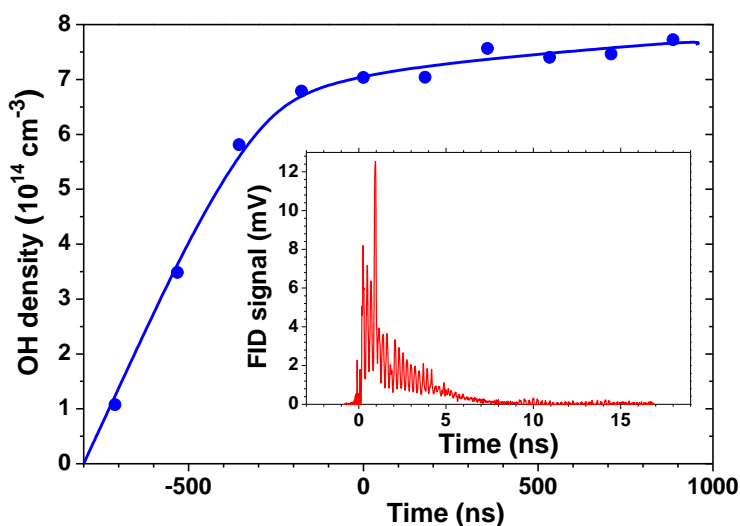

Fig. 4. Dynamics of OH-radical generation after shot pulse of UV radiation: points - treatment of the FID signals (insert) after series of ten NovoFEL excitation diagnostic pulses

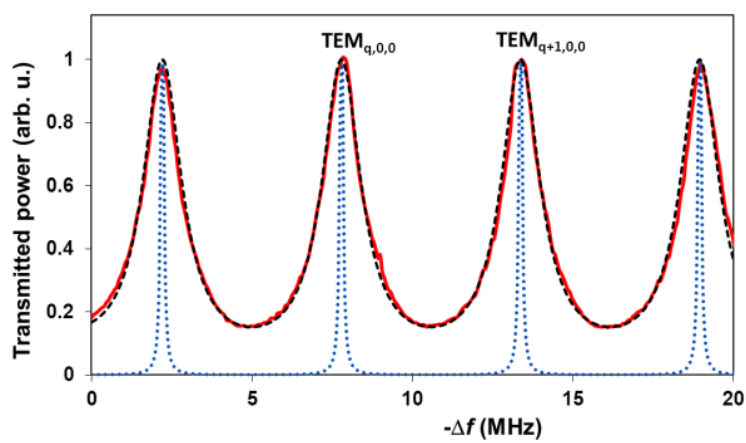

Fig. 5. Fine mode structure of the NovoFEL radiation measured by ultra-long waveguide vacuum mesh resonance FPI: solid red line - experiment, dash black line - instrumental function of the FPI, point blue line - reconstructed longitudinal mode structure of the NovoFEL

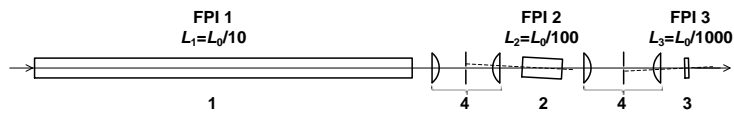

Fig. 6. Optical scheme of setup for one-mode filtration of the NovoFEL radiation: 1-3 - resonance FPI's (1 - ultralong waveguide vacuum mesh resonance FPI), 4 - optical isolators

This work was made with financial support of Russian Science Foundation (project 14-50-00080). The NovoFEL operation was supported by Ministry of Education and Science of The Russian Federation (project RFMEFI62117X0012).

\section{References}

1. Kulipanov, G. N., et al. Novosibirsk free electron laser - facility description and recent experiments // IEEE Transaction on Terahertz Science and Technology. 2015. V. 5, No. 5. P. 798-809.

2. Bolotin, V. P., et al. Quasi-continuous sub-millimeter optical discharge on Novosibirsk free electron laser: experiments and elementary theory // Proc. IRMMW-THz-2005. 2005. Williamsburg. USA. P. 126-127.

3. Kubarev, V. V. Dynamics of the $\mathrm{THz}$ optical discharge // Proc. IRMMW-THz-2014. 2014. Tucson. USA. T2-A-16.2.

4. Kubarev, V. V., Getmanov, Ya. V., Shevchenko, O. A Self-oscillations in terahertz laser discharge: Nature, param- eters, and suppression // Proc. IRMMW-THz-2016. 2016. Copenhagen. Denmark, DOI:10.1109/IRMMWTHz.2016.7759003.

5. Kubarev, V. V., Getmanov, Ya. V., Shevchenko, O. A., Koshlyakov, $P$. V. Threshold conditions for terahertz laser discharge in atmospheric gases // Journal of Infrared, Millimeter, and Terahertz Waves. 2017. V 38, No. 6. P. 787-798.

6. Kubarev, V. V., Getmanov, Ya. V., and Shevchenko, $O$. A. Continuous point-like high-temperature laser discharge produced by terahertz free //AIP Advances. 2017. V. 7. P. 095123-(1-8).

7. Kubarev, V., Chesnokov, E., Koshlyakov, P. Ultrafast high-resolution $\mathrm{THz}$ time-domain spectroscopy // Proc. IRMMW-THz-2012. 2012. Wollongong. Australia. Thu-C2-4.

8. Kubarev, V., Chesnokov, E., Koshlyakov, P. Terahertz free induction decay of molecular transitions: direct observation and practical use // Proc. TERA-2012. 2012. Moscow. Russia. P. 68-69.

9. Chesnokov, E. N., Kubarev, V. V., Koshlyakov, P. V., and Kulipanov, G. N. Direct observation of the terahertz optical free induction decay of molecular rotation absorption lines in the sub-nanosecond time scale // Appl. Phys. Lett. 2012. V. 101. P. 131109-(1-4).

10. Kubarev, V. V., Chesnokov, E. N., and Koshlyakov, $P$. V. One-pulse high-resolution $\mathrm{THz}$ time-domain spectroscopy: development and applications // Proc. IRMMW-THz2013. 2013. Mainz. Germany. Tu5-5.

11. Kubarev, V. V. Ultrafast high-resolution spectroscopy of separate NovoFEL pulses // Proc. IRMMW-THz2013. 2013. Mainz. Germany. Mo14-4.

12. Chesnokov, E. N., Kubarev, V. V., Koshlyakov, P. $V$., Kulipanov, G. N. Very long terahertz free induction decay in gaseous hydrogen bromide // Laser Phys. Lett. 2013. V. 10. P. 055701-055703.

13. Chesnokov, E. N., Kubarev, V. V., and Koshlyakov, $P$. $V$. Rotation commensurate echo of asymmetric molecules - Molecular fingerprints in the time domain // Appl. Phys. Lett. 2014. V. 105. P. 261107-(1-4).

14. Chesnokov, E. N., Kubarev, V. V., Koshlyakov, P. $V$., Getmanov, Ya. V., Shevchenko, O. A. Non-Faraday rotation of the free induction decay in gaseous $\mathrm{NO} / /$ Chemical Physics Letters. 2015. V. 636. P. 203-207.

15. Chesnokov, E. N., Kubarev, V. V., Koshlyakov, P. V., Chichinin, A. I., Getmanov, Ya. V., Shevchenko, O. A. The influence of magnetic field on the echo-like free induction decay in $\mathrm{NO}_{2} / /$ Chemical Physics Letters. 2016. V. 662. P. 62-66.

16. Chesnokov, E. N., Kubarev, V. V., Koshlyakov, P.V. Observation of the Formation of $0-\pi$ Pulses in Rotation Spectra of HCN and HBr // Physics Procedia. 2016. V. 84. P. $135-141$

17. Bragin A.V. et al. Superconducting solenoid for superfast THz spectroscopy // Physics Procedia. 2016. V. 84. P. $82-85$.

18. Chesnokov, E. N., Kubarev, V. V., Koshlyakov, P. $V$., and Fedorov, $V$. $V$. Heterodyne Method of Detection of Molecular Gas in the Terahertz Region Using the Beats Between Free Induction Decay Signals // IEEE Transactions on terahertz science and technology. 2017. V. 7, No. 2. P. 144-150.

19. Kubarev, $V . V$. Instabilities, coherency, and spectra of the NovoFEL radiation // EPJ Web of Conferences. 2017. V. 149. P. 05007(1-2).

20. Kubarev, V. V., Getmanov, Ya. V. NovoFEL as source of powerful ultramonochromatic tunable terahertz radiation // Proc. IRMMW-THz-2018. 2018. Nagoya. Japan. Fr-A2-1b-3 